\title{
Téoros
}

Revue de recherche en tourisme

\section{Le tourisme industriel et la ressource patrimoniale : en user sans en abuser}

\section{Gérard Beaudet et Jan O. Lundgren}

Volume 15, numéro 2, été 1996

Patrimoine industriel

URI : https://id.erudit.org/iderudit/1075020ar

DOI : https://doi.org/10.7202/1075020ar

Aller au sommaire du numéro

Éditeur(s)

Université du Québec à Montréal

ISSN

0712-8657 (imprimé)

1923-2705 (numérique)

Découvrir la revue

Citer ce document

Beaudet, G. \& Lundgren, J. O. (1996). Le tourisme industriel et la ressource patrimoniale : en user sans en abuser. Téoros, 15(2), 3-4.

https://doi.org/10.7202/1075020ar d'utilisation que vous pouvez consulter en ligne.

https://apropos.erudit.org/fr/usagers/politique-dutilisation/ 


\section{LE TOURISME INDUSTRIEL ET LA RESSOURCE PATRIMONIALE: EN USER SANS EN ABUSER}

\section{Gérard Beaudet et Jan O. Lundgren}

Le tourisme industriel n'est pas notweau. On peut en effet présumer que des touristes se sont toujours intéressés aux savoir-faire, aux liewx de production et ato produits de l'activite artisanale ou industrielle. Ce qui est nouveau cependant, c'est l'ampleur qu'a atteinte cette forme de tourisme. Le nombre de sites, de lieux et d'établissements de production industrielle accessibles s'est en effet considérablement accru et les visites industrielles sont désormais au programme de plusieurs vacanciers, touristes itinérants on touristes d'affaire.

Cet accroissement de la popularité du tourisme industriel peut être autribué à de multiples facteurs, dont la désindustrialisation massive et accellérée de nos sociétés dites avancées, la perte de contact avec les procédés de transformation et de fabrications traditionnels ainsi que les savoirs et les savoir-faire associes, la multiplication de procédés et d'équipements de production informatisés et robotises, woire la dematerialisation des procédés, ou encore l'auto. valorisation nationale, la mawaise conscience de certaines entreprises ou tout sinplement la capacité de récupération de tout filon le moindrement prometteur par une industrie touristique insatiable.

Le champ du tourisme industriel est aussi éclaté que les raisons de son explosion sont multiples. $I l$ englobe plus ou moins indis. tinctement les domaines de la technologie, de la culture scientifique, de l'anchéologie et de l'architecture industrielles, de la culture ouvriere, de la recherche et du développement de pointe, de la production artisanale, manufacturière, hatement wécanisée ou infornatisée. Ses liew sont tout autant diversifies, allant du site ne comportant que quelques vestiges a la grande entreprise où les visites s'effectuent sous haute surveillance, securite oblige, en passant par le moulin isolé a la campagne, les grandes agglonérations ouvrières ou les regions minieres.

Au Québec, le tourisme industriel est relativement nouveau, du moins en regard d'une organisation minimale de l'offre par la mise en valeur spécifique de lieux, de sites et de ressources. On peut toutefois en trower certaines manifestations des le siecle dernier, que ce soit lors d'expositions occasionnelles de machines industrielles ou à l'occasion de la frequentation de certaines réalisations constifuant des prouesses technologiques. Le pont Victoria, inauguré en 1854 et dès Lors considéré comme une mervelle de l'ingénierie, aurait ainsi ếté un des preniers produits touristiques industriels.

Le présent numéro de Téonos s'intéresse plus spécifiquement aux liens qui ont été établis entre le patrinoine industriel et le tourisme. en particulier au Québec. Marc Laplante y interoge, d'entréc de jeu, les ressorts de la rencontre entre le touriste ot l'womo faber. On y decouvre, en toile de fond, toute la richesse potentielle de cette renconte aux fondentens et aux formes multiples.

Ses observations wouvent un prolongentent dans les réflexions d'André Hut sur les conditions du tourisme industriel et dans la présentation de l'Écomusée du Fier monde par Rend Binette ainsi que de la formule de lécononusée par Helène Deslawiers. Alors que A. Hut souhate que la visile industrielle soit davantage whe veritable incursion dans lhistoire et la condition ouvrieres, $R$. Binette montre que depuis plus de quinze ans, cette mémoire ouvrière est demeurée une des assises d'un projet écomuséal en milieu industriel montrealais, cependant que H. Deslauriers insiste sur la présence indispensable de l'artisan porteur d'une ménoire au coeur de l'economusé, ce musée qui gagne sa vie. Mais, ces formes de mise en valeur sont-elles compatibles avec un tourisme de masse? On peut en douter. Elles n'en demeurent pas moins indispensables, justement peut-être parce qu'elles y résistent.

Les réflexions de André Hut trouvent par ailleurs un écho dans le texte de Gérard Beaudet dont la présentation des lieux et des modes de mise en valeur du patrinoine industriel québécois fait ressontir que, malgré la multiplication des sites industriels accessibles au public, le tourisme industriel demeure très embryonnaire. II conclut d'ailleurs sa présentation par une interrogation sur la capacité de l'interprétation d'accéder à la substance de ces lieux dans une société qui valorise si peu la culture scientifique. Mais, son anticle pose egalement de manière plus large le problème de l'adequation entre la ressource et sa mise en valeur.

C'est ce que font également a leur maniere les textes d'André Bédard sur le canal de Lachine et de Robert Shipley sur le canal Welland. Dans l'un et l'autre cas, on se rend compte que les enjeux de la conservation et de la mise en valeur du patrimoine industriel sont multiples et parfois contradictoires et qu'en ce dowaine rien n'est definitivement acquis, peu importe l'intérêt de la ressource. Quiconque suit lewolution du projet de mise en valew du canal de Lachine depuis une quinzaine d'années en conviendra wolontiers. 
Paul Fritz-Nemeth et Jan O. Lundgren prennent d'ailleurs prétexte d'une reconnaissance du potentiel patrimonial exceptionnel de l'heritage industriel d'une partie des Cantons de l'Est pour véfléchir sur le processus d'émergence de noweaux produits touristiques. Le territoire de référence est d'autant plus intéressant qu'il est fortement marqué par la grande villegiature du $X I X e$ siècle, qui est également un des fruits de la révolution industrielle. $O r$; on doit reconnaitre que ces grands domaines associées à la mise en valeur des ressources naturelles paysagères peuvent être des lieux de résistance au fourisme industriel et au modèle théorique. C'est notamment ce que montre la cession de l'emprise ferroviaire abandonnée aux riches riverains saisonniers du lac Massawippi, ce qui a irrémédiablement compromis un projet de piste cyclable.

L'incursion d'autres régions du Québec dans le champ du tourisme industriel patrimonial est parfois spectaculaire. C'est ce dont témoignent le texte de Guy Perreault sur le tourisme minier en AbitibiTémiscamingue et celui de Normand Cazelais, Benout Gauthier et Jean-Marc Carpentier sur la Cúté de l'Énergie de Shawinigann.

Ces lieux d'interprétation constituent, avec la pulperie de Chicoutimi, malheureusemnent ravagée en cette mi-juillet par la crue spectaculaire de la rivière du même nom, les principaux foyers d'émergence de mémoires régionales fortement imprégnées d'un heritage industriel parfois lourd à porter: On mise beaucoup, en ces régions eprouvées par la restructuration de nos économies, sur les retombées de ces grands projets. Les prochaines années sauront nous indiquer si les attentes des promoteurs etaient fondes et si le patrimoine industriel y a trouvé son compte.

Finalement, Marie-Claude Robert esquisse un projet de cartographie des sites industriels québécois d'avant 1950 qui sont accessibles au public. Cette initiative de l'Association québécoise pour le patrimoine industriel aura évidemment l'intérêt de révéler l'étendue de ce champ patrinonial. Mais elle pourrait également permettre la constitution de circuits régionaux de découverte. Il y a là une richesse parfois exceptiomelle qui reste très mal connue. Saura-ton l'exploiter judicieusement ou retomberat-on dans les abus qui ont justement caractérisé la révolution industrielle? Bien malin qui saurait trancher.

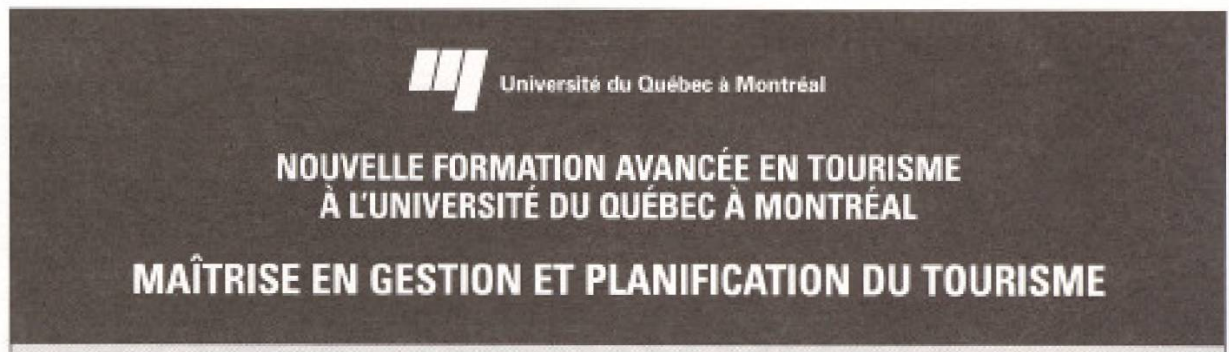

Ce programme sera offert à compter de la session d'hiver 1997.

Il est sous la responsabilité conjointe du département d'études urbaines et touristiques et du département des sciences administratives de l'UQAM.

\section{Objectifs}

De type professionnel, le programme de maitrise en gestion et planification touristiques poursuit un double objectif général: former, dans le secteur touristique, des gestionnaires de haut niveau aptes à gérer l'infrastructure de services de façon professionnelle; former des spécialistes de la planification stratégique capables de concevoir des stratégies de développement tenant compte de l'environnement naturel, culturel, économique, juridique, administratif et des ressources matérielles, financières et humaines disponibles.

Le programme vise les quatre objectifs spécifiques suivants: l'acquisition des connaissances; le développement des aptitudes et des compétences; la préparation ả des fonctions de responsabilité dans les secteurs privé et public; l'ouverture aux dimensions internationales.

\section{Conditions d'admission}

1) Le candidat doit détenir un diplôme de premier cycle d'une université reconnue ou un diplôme jugé équivalent dans l'une des disciplines suivantes: sciences de l'administration; sciences sociales: notamment, sociologie, anthropologie, géographie, politique, économie; discipline reliée au tourisme, par exemple: gestion touristique, urbanisme ou aménagement du territoire, récréologie, loisir, histoire de l'art.

2) Et avoir une expérience de travail jugée pertinente d'au moins deux ans.

Le candidat qui ne détient pas de diplôme de premier cycle doit posséder les aptitudes, la formation de base et une expérience pratique d'au moins quatre ans comme professionnel dans une entreprise touristique. Le candidat qui ne possède pas la formation scolaire de base pourrait être invité à suivre des cours préparatoires. Celui dont la connaissance en méthodes quantitatives sont jugées insuffisantes devra réussir, avant d'entreprendre la première session, le cours d'appoint MBA8000 - Éléments de méthades analytiques.

De plus, le candidat doit posséder une moyenne cumulative d'au moins 3.2 sur 4.3 ou l'équivalent, fournir deux lettres de référence; si nécessaire, une entrevue pourra être exigée.

Grade

Maître ès-sciences (M.Sc.)

Crédits

Ce programme de type professionnel comporte 45 crédits.

\section{Renseignements}

Pour plus de renseignements ou pour obtenir un formulaire d'admission, prière de vous adresser au numéro de téléphone suivant: (514) 987-3000, poste 4750 ou par télécopieur: (514) 987-7827. 